\title{
Repeatability and Accuracy of Bone Cutting and Ankle Digitization in Computer-Assisted Total Knee Replacements
}

\author{
Kevin B. Inkpen ${ }^{1}$, Antony J. Hodgson ${ }^{1}$, Christopher Plaskos ${ }^{1}$, \\ Cameron A. Shute ${ }^{1}$ and Robert W. McGraw ${ }^{2}$ \\ Departments of ${ }^{1}$ Mechanical Engineering and ${ }^{2}$ Orthopaedics \\ University of British Columbia, Vancouver, BC, Canada \\ Email: ahodgson@mech.ubc.ca
}

\begin{abstract}
In conventional total knee replacement (TKR) surgery, a significant fraction of implants have varus/valgus alignment errors large enough to reduce the lifespan of the implant, so we are developing a more accurate computerassisted procedure aimed at reducing the standard deviation (SD) of the implant's frontal alignment to under $1^{\circ}$. In this study we measured the contributions to overall alignment error of two steps in our proposed procedure: ankle digitization and manual bone cutting. We introduce a new digitizing probe that quickly and robustly locates the midpoint between the ankle malleoli. Based on repeated measurements on 8 cadavers by 6 operators (318 measurements), we estimate that the new probe introduces only $0.15^{\circ}$ (SD) of variability into the definition of the tibial mechanical axis in the frontal plane. We also measured the accuracy and repeatability with which surgeons can implement a bone cut using conventional cutting guides to see if conventional cutting techniques are sufficiently accurate. A total of 53 tibial plateau, distal and anterior/posterior $(\mathrm{A} / \mathrm{P})$ femoral cuts approximated primary and revision TKR resections. In 20 test cuts on cadaver bone made by 2 expert TKR surgeons, we found a SD of $0.37^{\circ}$ (bias of $0.29^{\circ}$ ) in the varus/valgus difference between the guide orientation and the implant orientation before cementing, but in 23 additional cuts performed by four less-experienced surgeons, the SD was $0.83^{\circ}$ (bias of $0.31^{\circ}$ ). Ten A/P femoral cuts showed similar trends. We conclude that, in the hands of an experienced surgeon, our current technique (based on our previously-reported non-invasive hip centre locating technique [Inkpen 1999b], robust ankle digitization and manual cutting using computerguided cutting guides) can approach our target alignment variability goal of a SD less than $1^{\circ}$.
\end{abstract}

\section{Introduction}

We are developing computer-assisted total knee replacement (TKR) tooling that eliminates intramedullary rods and improves alignment accuracy without introducing additional imaging requirements (such as preoperative computed tomography scans) or invasive procedures (such as bone pins remote to the operating site). Varus/valgus alignment error of $3^{\circ}$ has been strongly correlated with aseptic loosening [Jeffery 1991]. In a meta-analysis of 10 published studies involving 1373 knees, we estimate the SD of varus/valgus alignment to be $2.6^{\circ}$ on the overall limb and $1.9^{\circ}$ each for the distal femoral and tibial plateau cuts [Inkpen 1999a]. To ensure that upwards of $95 \%$ 
of all computer-assisted procedure result in overall alignment lying within this critical $3^{\circ}$ window, it is necessary to reduce the $\mathrm{SD}$ of the overall procedure to approximately $1^{\circ}$.

In the computer assisted TKR technique developed by Leitner et al [Leitner 1997], the mechanical axis is defined intraoperatively using a 3D optoelectronic localizer. They find the hip centre by tracking a marker at the distal femur in a reference frame rigidly attached to the pelvis (using a bone pin) as the femur is moved through its range of motion, taking the centre of the best fitting sphere to the femoral path as the hip centre. Krakow et al [Krackow 1999] use a similar technique for the hip but eliminate the bone pin at the pelvis by simply holding the patient steady during the femoral motion. In early testing we have achieved precise and accurate hip centre location using a new non-invasive hip tracker and, although more testing on a variety of patients is ongoing, we estimate that the femoral mechanical axis can be located intraoperatively with a SD of $0.05^{\circ}$ [Inkpen 1999a].

Krakow et al use a conventional point probe to digitize the medial and lateral malleoli, and the ankle centre point is then defined as the intersection of this malleolar axis and a digitized unit vector representing the surgeons' estimate of the best centre along the anterior portion of the ankle. Leitner, despite the complexity of the ankle joint complex, uses the same sphere-fitting algorithm applied to the hip to find the ankle centre. They track a bone pin marker inserted in the calcaneus in a coordinate frame pinned to the tibia. In an attempt to validate this determination of a kinematic ankle centre, Leitner compared the sphere fitting method with direct digitization on a plastic skeleton leg. Although not directly comparable to a human study, digitizing the mechanical centres of the skeleton showed better repeatability over the sphere fitting method $\left(0.29^{\circ}\right.$ vs. $\left.0.49^{\circ} \mathrm{SD}\right)$. We also have found poor repeatability with the motion tracking technique even when a bone pin marker is used in the calcaneus [Inkpen 1999a, Inkpen 1999b]. Even though digitization is more repeatable than motion-tracking techniques at the ankle, the contribution of variance is still large in comparison with that found at the hip. Using a conventional point probe, a user who is asked to digitize an ankle malleolus could select any point within an area of radius $\sim 3-5 \mathrm{~mm}$ and still consider the point to be 'on' the malleolus. Although a careful operator may be very precise using this method, we would not expect it to be robust across different operators and patients. It is also not clear that a motion tracking and sphere fitting method without realistic weight bearing load gives a biomechanically correct ankle centre. A passively manipulated ankle acts about a single talocrural joint axis [Leardini 1999], although inversion/eversion torques generate motion at the subtalar joint, so the ankle complex can act more like a biaxial joint [van den Bogert 1994]. Given the practical problems of simulating and tracking weight bearing ankle motion intraoperatively, we propose using a robust digitizing probe to precisely locate the malleoli, allowing the traditional ankle centre estimate of the midpoint between the malleoli to be used in the computer-assisted technique.

Finally, once the patient's mechanical axis is registered, cutting planes for the desired limb alignment can be calculated and conventional cutting guides fitted with markers can be manually adjusted to the correct pose [Leitner 1997][Krackow 1999]. For cemented TKR where the surface quality from a conventional bone saw is sufficient, the question is whether errors due to conventional cutting are large enough to warrant more sophisticated bone cutting tools such as robotic guidance of the bone 
saw or robotic machining. One study of cuts made in balsa wood blocks shows flexion/extension SD of $0.25^{\circ}$ with a bias of $1^{\circ}$ towards flexing of the saw blade up and away from the guide surface [Otani 1993]. Although several studies have been made of the bone surface quality and roughness after cutting, we could not find any published studies on overall implant orientation error in the frontal plane compared to the guide setting.

\section{Methods}

\subsection{Ankle Digitizing Probe Design and Testing}

Probe Design: In making the traditional estimate of the ankle centre based on the malleoli, precision and robustness could be improved if the digitizing probe only fit onto the malleolus in a certain way, forcing the user to place the probe in a smaller range of positions. As the malleoli are roughly spherical in shape (even indistinct malleoli can often be palpated well enough to find some amount of spherical protrusion), the ankle probe (Figure 1a) contacts the anatomical feature with three 7.8 $\mathrm{mm}$ diameter balls spaced evenly on a common $12.8 \mathrm{~mm}$ radius about the probe centreline, with all 3 contact ball centres lying in a plane normal to the centreline. A plunger is free to move along the probe centreline and contacts the feature with a normal planar face. When the probe is pressed against a roughly spherical feature, the three contact balls force the centreline of the probe to pass through the sphere centre and the plunger face contact point lying on the sphere centreline is known (Figure 1b). Unless used in a completely careless way, this design repeatably guides the plunger contact point to the extreme medial and lateral surfaces of the malleoli.
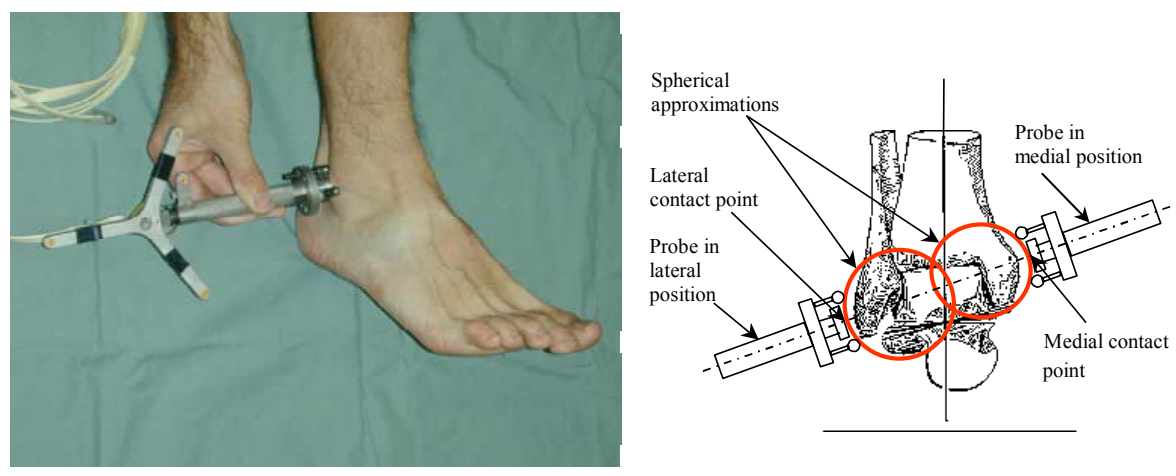

Figure 1. Ankle probe in use (a) and frontal plane view of both measurement positions (b).

Study design and data analysis: To simulate conditions in a computer-assisted TKR, sets of 21 to 30 repeated measurements of the ankle centre were made by 6 different operators on 8 different embalmed cadavers for a total of 318 ankle centre measurements. To estimate intra-observer repeatability on a variety of patients, we report the standard deviation (SD) of 30 ankle centre measurements by a single operator on 7 different embalmed cadavers. To show the probe's robustness when used by different operators, three additional operators each made 21 to 30 repeated 
measurements on one of these specimens and we report the total range spanning the $95 \%$ confidence limits of each individual operator. To show the relation between the characteristic ankle point found with the probe and the centre of area for the articular surface, two additional operators each made 20 measures of an additional specimen, after which we dissected the talocrural joint, digitized the tibial mortise and calculated its centre of area.

Measurement techniques: All measurements were recorded relative to a threeemitter triangular local reference frame $(120 \mathrm{~mm}$ on a side) pinned rigidly to the proximal tibia and nominally aligned with the body planes. We used a Flashpoint 5000 localizer (Image Guided Technologies, Boulder, CO, USA) for all measurements, which has a typical accuracy of $\sim 0.5 \mathrm{~mm}$ [Chassat 1998]. We removed indentations in the skin by massaging the tissue overlying the malleoli between measurements to prevent the probe contact balls from repeatedly falling into the same position.

\subsection{Bone Cutting Errors}

Study design: For this study we define bone cutting error as the difference in orientation between the cutting guide and the implant, described as two rotations. The first is in the plane of the face of the cutting guide facing the surgeon (labeled varus/valgus for distal femoral and tibial plateau cuts, and internal/external rotation for $\mathrm{A} / \mathrm{P}$ femoral cuts), and the second is about a nominally mediolateral (ML) axis defined by the intersection of the front face plane and the cutting plane of the guide (labeled flexion/extension for distal femoral and tibial plateau cuts, and anterior/posterior deviation for $\mathrm{A} / \mathrm{P}$ femoral cuts).

Two expert TKR surgeons, three orthopaedic residents with TKR experience and a foreign orthopaedic surgeon without TKR experience made cuts simulating typical practice on five fresh frozen human femora and eight tibia. Several cuts were made on each specimen, approximating primary and revision bone resections, for a total of 53 cuts. The specimens had no obvious abnormalities in bone quality. Cutting guides, a conventional pneumatic oscillating bone saw (CPS \#1535), and saw blades supplied by Johnson \& Johnson (Raynham, Mass. USA). We used open guides for 33 cuts and slotted guides for the remainder. 8 cuts were made with a $0.8 \mathrm{~mm}$ thick, 75 $\mathrm{mm}$ long blade (Synvasive 'Stablecut' \#11-0470) and the remainder were made with $1 \mathrm{~mm}$ thick, $90 \mathrm{~mm}$ long blades (Johnson \& Johnson \#26-6050) renewed at the surgeon's request.

To simulate orientation of the components after placement on the cut bone, we made dummy components from flat aluminum plate by copying the profile of the tibial trays and the distal footprints of the femoral components in a Johnson \& Johnson PFC implant series. For each test cut, the surgeon pinned the guide to the specimen and we measured the front face and cutting surfaces of the guide to provide the reference frame for reporting errors in varus/valgus (internal/external rotation for $\mathrm{A} / \mathrm{P}$ cuts) and flexion/extension (anterior/posterior deviation for $\mathrm{A} / \mathrm{P}$ cuts). The surgeon then made the cut and did any trimming and checking as would normally be done in TKR. We then re-measured the guide, placed a dummy implant on the cut bone surface to simulate placement of an implant with proper bone coverage, and measured the plane of the dummy implant. 
Measurement techniques and data analysis: All measurements were made using the Flashpoint 5000 localizer (described above). To determine the variability associated with placing a dummy implant on a bone cut and measuring its orientation, we made 30 repeated placements and measurements on a single bone cut. The resulting $\mathrm{SD}$ was $<0.10^{\circ}$ for varus/valgus errors and $<0.17^{\circ}$ for flexion/extension errors (95\% confidence). For test cuts, we computed the mean and SD of all cutting errors in each direction. We are continuing to enroll surgeons in this study, so until we have a sufficient number to represent the general surgical population and until more cuts are made with slotted guides, we will not try to extract the effects of guide style, or type of cut.

\section{Results}

\subsection{Ankle Digitizing Probe Results}

The average SD of ankle centre location for any one set of repeated measurements is $0.75 \mathrm{~mm}$ in the mediolateral (ML) direction and $1.12 \mathrm{~mm}$ in the anteroposterior (AP) direction (indicating that the new probe will give a result within $\pm 1.9 \mathrm{~mm}$ (ML) and $\pm 2.9 \mathrm{~mm}$ (AP) $95 \%$ of the time, Figure 2). This corresponds to an angular variability of $0.15^{\circ} \mathrm{SD}$ in varus/valgus and $0.2^{\circ} \mathrm{SD}$ in flexion/extension when registering a short (375 mm) tibial mechanical axis. The maximum range of a single operator on a single specimen is $6.5 \mathrm{~mm}$ (ML) and $10 \mathrm{~mm}$ (AP), giving a maximum tibial axis range of $1.0^{\circ}$ varus/valgus and $1.5^{\circ}$ flexion/extension.

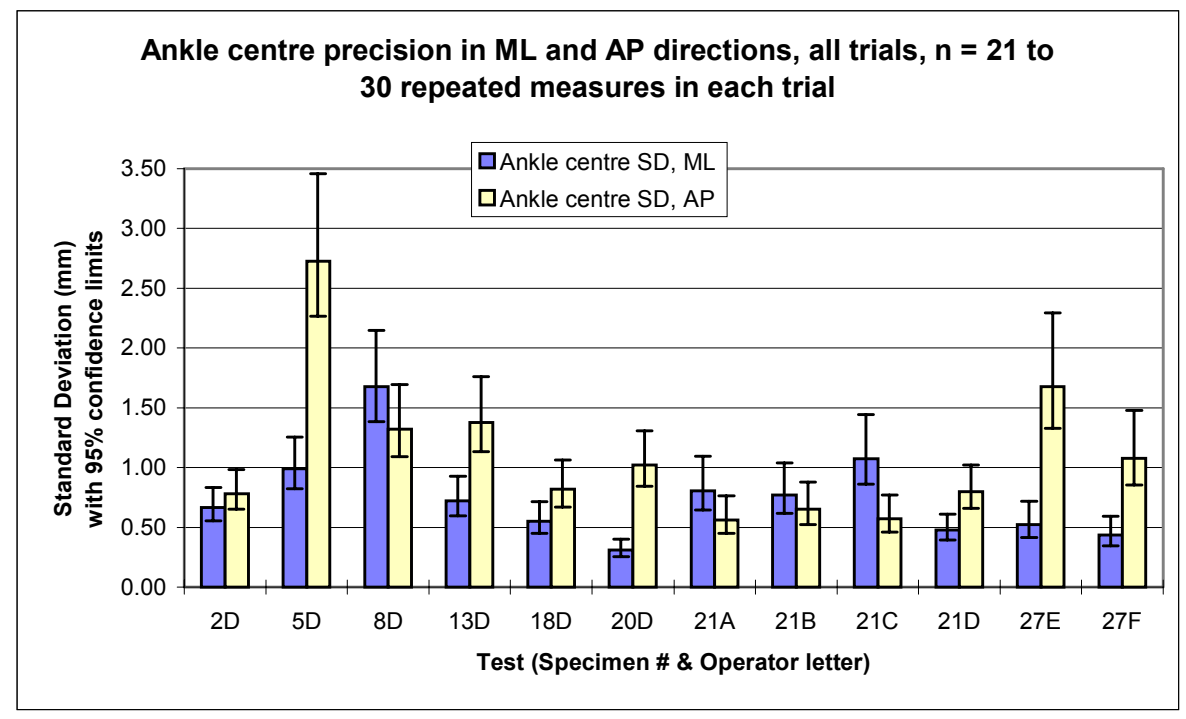

Figure 2. Range of variation in estimated ankle centre locations in the mediolateral (ML) and anteroposterior (AP) directions based on multiple specimens and operators.

A truly robust method would have no significant differences between the operator means. All means are within $0.8 \mathrm{~mm}$ in both AP and ML directions (Figure 3) and 
the range of the $95 \%$ confidence limits of the means is $1.6 \mathrm{~mm}(\mathrm{ML})$ and $1.3 \mathrm{~mm}$ (AP).
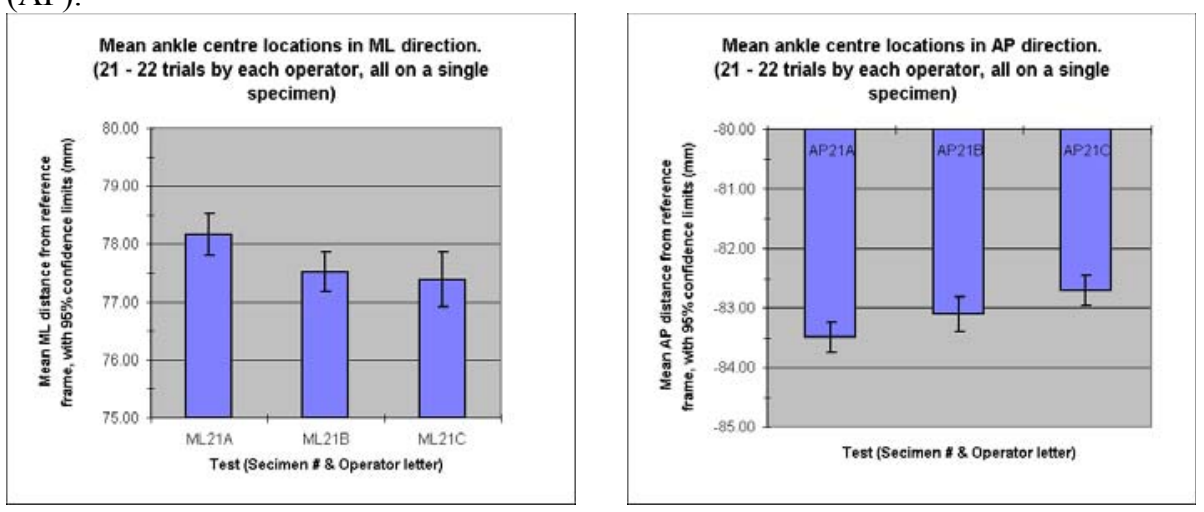

Figure 3. Interoperator differences in estimated ankle centre locations in the ML and AP directions on a single specimen (ankle centre locations are relative to a local reference frame pinned rigidly to the proximal tibia and nominally aligned with the body planes).

On the one specimen available for dissection, 2 operators both obtained mean results within 2 to $3 \mathrm{~mm}(\mathrm{ML})$ and 2 to $5 \mathrm{~mm}(\mathrm{AP})$ of the talocrural articular surface centroid (Figure 4).

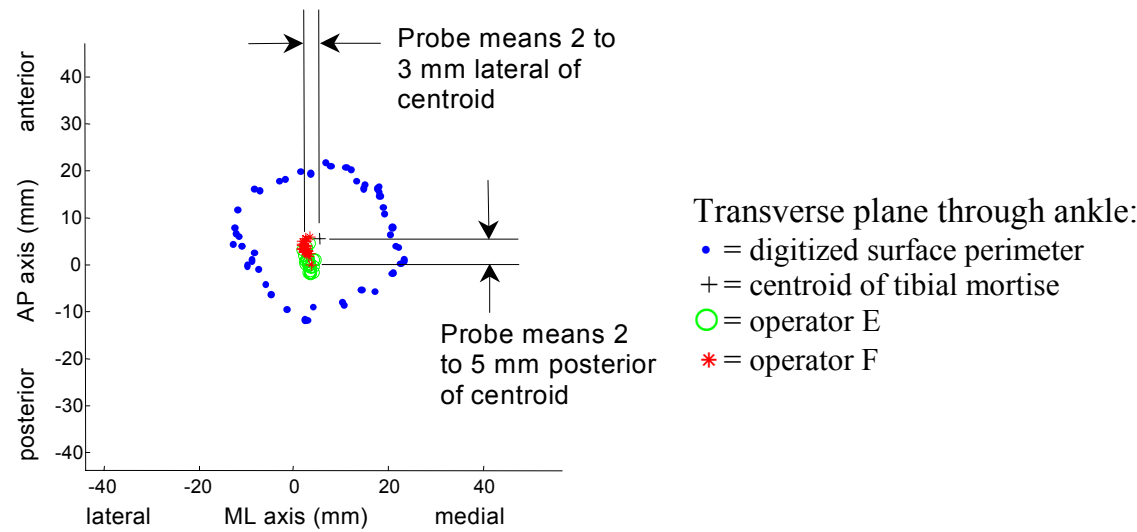

Figure 4. Comparing mean ankle centre locations, obtained by two operators, with centroid of tibial mortise, obtained by direct digitization, all on one specimen.

\subsection{Bone Cutting Results}

Both the guide movement and implant errors follow a normal distribution (checked using normal scores plots). Based on the actual variances, the test has $95 \%$ power to detect an implant alignment error of $0.4^{\circ}$ in varus/valgus and $0.7^{\circ}$ in flexion/extension. The mean cutting error in the frontal plane is about $0.3^{\circ}$ degrees in the varus/valgus direction for both surgeon types (Figure 5). Precision in varus/valgus is within a $\mathrm{SD}$ of $0.54^{\circ}$ for the experts and $1.25^{\circ}$ for the less experienced 
surgeons (95\% confidence); guide movement accounts for about $30 \%$ of the expert surgeons' variance and $34 \%$ of the less experienced surgeons' variance.
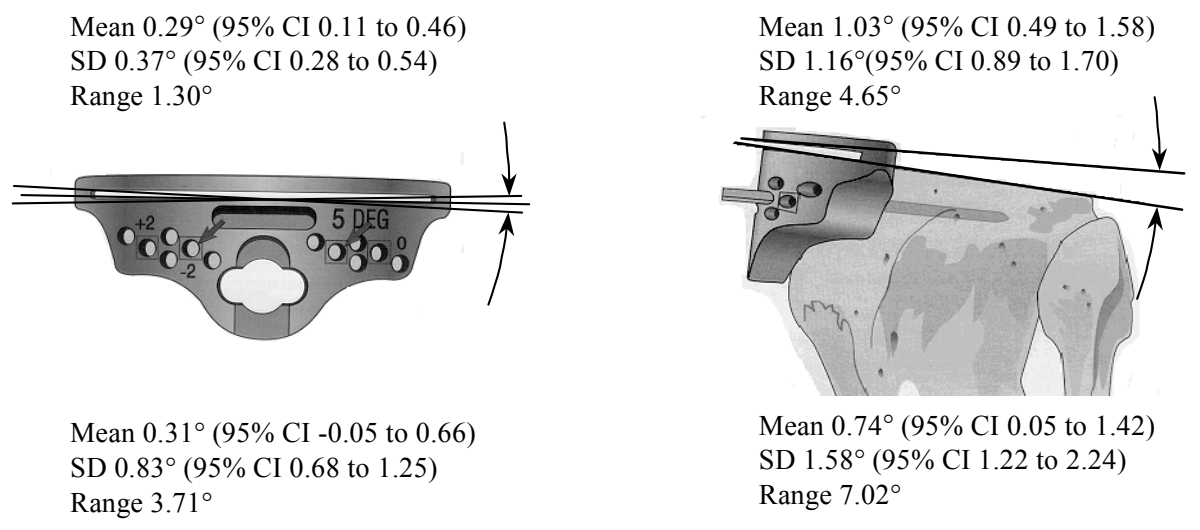

Figure 5. Varus/valgus (left) and flexion/extension (right) implant error results for 43 bone cuts: 20 made by 2 expert surgeons (top) and 23 by 2 less experienced surgeons (bottom).
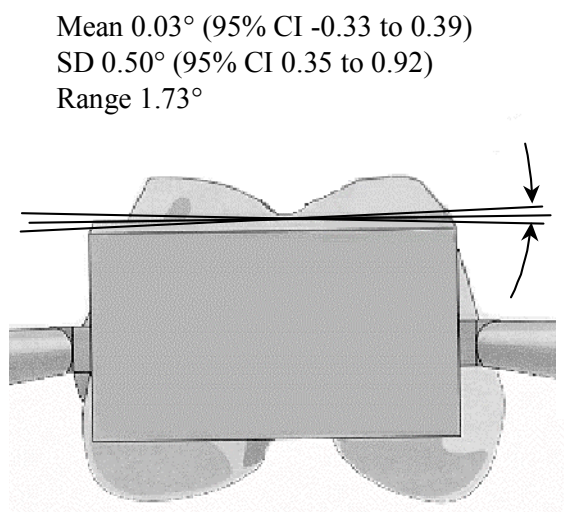

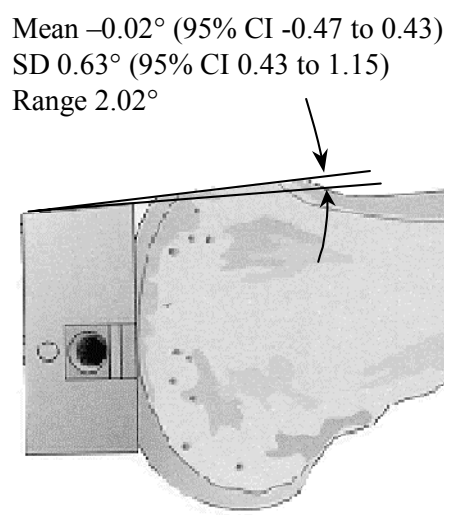

Figure 6. Internal/external rotation (left) and anterior/posterior deviation (right) implant error results for 10 bone cuts.

In flexion/extension, there is a bias towards flexing of the saw blade up and away from the guide surface of over $1^{\circ}(\mathrm{P}=0.0006)$ for the expert surgeons. The greatest flexion/extension errors in the current study occurred on large specimens where the saw blade did not reach the end of the cut before the saw body hit the guide, requiring the cut to be finished off 'freehand' at the far cortex. Precision in flexion/extension for both surgeon groups is within a SD of $2.24^{\circ}$, where guide movement accounts for about $37 \%$ of this variance. The measurement variance in either direction accounts for only about $1 \%$ of the observed variance.

\footnotetext{
* Positive mean rotations are counter-clockwise about axes pointing out of the page.
} 
The results of ten $\mathrm{A} / \mathrm{P}$ femoral cuts executed by an expert surgeon (six cuts) and a resident (four cuts) on four femora are shown in Figure 6. Error in internal/external rotation (about the femoral mechanical axis) averages to a SD of $0.50^{\circ}\left(0.34^{\circ}\right.$ for the expert and $0.67^{\circ}$ for the resident). In the sagittal plane, angular deviation of the resected bone plane relative to the cutting guide surface resulted in a SD of $0.63^{\circ}$ $\left(0.70^{\circ}\right.$ for the expert and $0.50^{\circ}$ for the resident $)$.

\section{Discussion}

\subsection{Ankle Digitizing Probe}

We found excellent repeatability in using digitization to locate a characteristic point within the ankle joint complex. Our ankle probe only introduces a frontal plane SD of $0.15^{\circ}$, which compares favourably to Leitner's digitization result of $0.29^{\circ}$. A future repeatability study that will compare our probe to an alternative method such as using a point probe to digitize the malleoli is also required to validate the robustness of this new design. We plan to do this in vivo because the skin dimpling encountered in cadaveric studies artificially decreases the repeatability of locating the same points with the point probe. We are currently working on developing an ankle centre location protocol that uses the talocrural and subtalar joint axes to define a biomechanically meaningful centre. More specimens need to be measured and dissected to show that a reliable relationship between the digitized point and a biomechanically relevant ankle centre point can be established.

\subsection{Bone Cutting}

In contrast to the excellent repeatability found at the ankle, bone cutting introduces a significantly higher contribution to the overall alignment variance. While the expert surgeons have limited their varus/valgus errors to under $1^{\circ}\left(0.37^{\circ} \mathrm{SD}\right)$, errors as high as $2^{\circ}\left(0.83^{\circ} \mathrm{SD}\right)$ were found with the less experienced surgeons. This corresponds to a $13 \mathrm{~mm}$ ML error in ankle registration, which is much higher than errors experienced with the robust ankle probe.

While only ten $\mathrm{A} / \mathrm{P}$ femoral cuts were made, the internal/external rotation errors follow similar trends to those found in varus/valgus. The expert surgeon's SD in internal/external rotation error is almost equal to that in varus/valgus $\left(0.34^{\circ}\right.$ and $0.37^{\circ}$ respectively) and is half that of the less experienced surgeon. However, we do not see the sagittal plane bias of the saw blade flexing up and away from the guide surface as with flexion/extension errors. This flexion/extension sagittal plane bias is similar to that found by Otani [Otani 1993]. It may be possible that the amount of bone resected in an $\mathrm{A} / \mathrm{P}$ femoral cut is not enough to cause the blade to flex away from the guide surface and there is no need for any freehand trimming.

Although we have noted marked differences in repeatability in bone cutting between the two expert surgeons and the four surgeons with less TKR experience, we have not yet studied enough surgeons to make a statistically valid argument that this is the case. As mentioned above, we are continuing to enroll surgeons of various experience levels in this study and hope soon to be in a position to test the effect of experience on repeatability in bone cutting. These results are also based on the use of 
both slotted and open guides; here too we expect to be able to test for differences in performance when using different guide types once we have enrolled more surgeons into the study. Finally, although the surgeons who have participated have commented that the cutting process on the benchtop is essentially identical to that in the operating room, we do not yet have any OR data to support this impression. As our system moves closer to clinical implementation, we will be in a position to test this hypothesis.

\subsection{Cumulative Repeatability}

There are numerous contributors to variability in our computer-assisted TKR procedure; these include variance and bias in estimating the hip, knee and ankle centres, setting the correct cutting guide pose, cutting the bone, and cementing the implant into place. To date, we have only measured values for the hip and ankle and for the bone cuts. Assuming that the knee measurements will contribute roughly the same variability as those at the hip and ankle, and assuming that the angular precision of locating the cutting guides will be similar to that of reading the planar probe, we estimate the overall repeatability of our proposed procedure by summing the variances of the various measurements. When we do this using the data for all six surgeons, we find that our predicted repeatability, before cementing, is roughly $1^{\circ}$ (SD). The cutting errors, particularly for the less experienced surgeons, contribute the greatest fraction to the overall variance $(\sim 90 \%)$, so further development of this procedure should focus on the cutting issue and the precision of the cementing process, which is currently unknown.
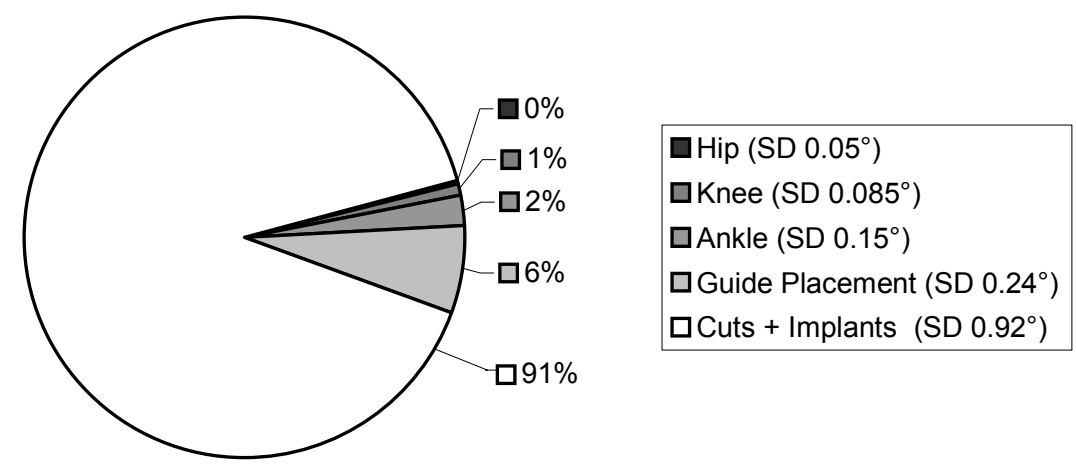

Figure 7. Contributions to overall varus/valgus variance in computer assisted TKR procedure. 


\section{Conclusions}

We conclude that even inexperienced surgeons can likely achieve a SD within $1.5^{\circ}$ for overall varus/valgus alignment in computer-assisted TKR using our proposed procedure consisting of a non-invasive hip centre identification technique, a robust ankle digitizing technique and a conventional cutting technique based on a computerguided cutting guide. Expert surgeons exhibit significantly better cutting precision, so we expect their overall precision to be better $\left(<1.2^{\circ}\right)$. In flexion/extension, alignment precision will be worse due to greater cutting errors in this direction.

\section{Acknowledgements}

We thank Drs. Thomas Oxland of the Division of Orthopaedic Engineering Research, Vancouver Hospital, and Vlad Stanescu, UBC Dept. of Anatomy, for their assistance.

\section{References}

1. Chassat F, Lavallee S: Experimental Protocol of Accuracy Evaluation of 6-D Localizers for Computer-Integrated Surgery: Application to Four Optical Localizers. In: Wells, W; Colchester, A; Delp, S. (eds): Medical Imaging and Computer Assisted Intervention (MICCAI'98). Lecture Notes in Computer Science Vol. 1496, Springer-Verlag 1998, pp. 421-430.

2. Jeffery RS, Morris RW, Denham RA: Coronal Alignment After Total Knee Replacement. J. of Bone and Joint Surgery (British Ed.) 73-B, 1991, pp. 709-714.

3. Inkpen, KB: Precision and Accuracy in Computer-Assisted Total Knee Replacement. Master's thesis, Dept. of Mechanical Engineering, University of British Columbia, Vancouver BC Canada. 1999a.

4. Inkpen KB, Hodgson AJ: Accuracy and Repeatability of Joint Centre Location in Computer-Assisted Knee Surgery. In: Taylor C, Colchester A (eds): Medical Imaging and Computer Assisted Intervention (MICCAI'99). Lecture Notes in Computer Science Vol. 1679, Springer-Verlag 1999b, pp.1072-1079.

5. Krackow, K.A., Bayers-Thering, M., Phillips, M.J. (1999). A New Technique for Determining Proper Mechanical Axis Alignment During Total Knee Arthroplasty: Progress Toward Computer-Assisted TKA. Orthopedics., 22(7), 698-702.

6. Leardini, A., O'Connor, J.J., Catani, F., \& Giannini, S. (1999). Kinematics of the human ankle complex in passive flexion; a single degree of freedom system. J.Biomech., 32(1), 111-118.

7. Leitner F, Picard F, Minfelde R, Schulz H-J, Cinquin P, Saragaglia D: Computer Assisted Knee Surgical Total Replacement. In: Troccaz J, Grimson E, Mosges R (eds): CVRMedMRCAS '97. Lecture Notes in Computer Science Vol. 1205, Springer-Verlag 1997, pp. 627-638.

8. Otani T, Whiteside LA, White SE: Cutting Errors in Preparation of Femoral Components in Total Knee Arthroplasty. Journal of Arthroplasty, Vol 8, No 5, Oct. 1993 pp. 503-510.

9. Bogert, A.J. van den, Smith GD, Nigg BM. In Vivo Determination of the Anatomical Axes of the Ankle Joint Complex: an Optimization Approach, Journal of Biomechanics, Vol. 27(12), 1477 - 1488, 1994. 